\title{
Malignancy following heart transplantation: differences in incidence and prognosis between sexes - a multicenter cohort study
}

Carlos Minguito-Carazo ${ }^{1}$, Manuel Gómez-Bueno ${ }^{2,3}$, Luis Almenar-Bonet ${ }^{4}$, Eduardo Barge-Caballero ${ }^{3,5,6}$, Francisco González-Vílchez ${ }^{7}$, Juan F. Delgado-Jiménez ${ }^{3,8}$, José María Arizón del Prado ${ }^{9}$, Iago Sousa-Casasnovas ${ }^{3,10}$, Sónia Mirabet-Pérez ${ }^{11}$, José González-Costello $^{12}$, José Manuel Sobrino-Márquez ${ }^{13}$, Félix Pérez-Villa ${ }^{14}$, Beatriz Díaz-Molina $^{15}$, Gregorio Rábago Juan-Aracil ${ }^{16}$, Teresa Blasco-Peiró ${ }^{17}$, Luis De la Fuente Galán ${ }^{3,18}$, Iris Garrido-Bravo ${ }^{3}$, Luis García-Guereta ${ }^{20}$, Manuela Camino ${ }^{21}$, Dimpna C. Albert-Brotons ${ }^{22}$, Javier Muñiz ${ }^{3,6,23}$, Maria G. Crespo-Leiro ${ }^{3,5,6,23}$.

Cardiology Department, Complejo Asistencial Universitario de León, León, Spain.

2 Heart Failure, Heart Transplant and Pulmonary Hypertension Unit, University Hospital of Puerta de Hierro, Madrid, Spain.

3 Centro de Investigación Biomédica en Red de Enfermedades Cardiovasculares (CIBERCV), Instituto de Salud Carlos III, Madrid, Spain.

4 Heart Failure and Transplant Unit, Cardiology Department of Hospital Universitari i Politècnic La Fe, Valencia, Spain.

5 Cardiology Department of Complexo Hospitalario Universitario de A Coruña (CHUAC) Sergas, A Coruña, Spain.

6 Instituto de Investigación Biomédica de A Coruña (INIBIC), A Coruña, Spain.

7 Advanced Heart Failure and Transplant Unit, Hospital Universitario Marqués de Valdecilla, Santander, Spain.

8 Heart Failure and Transplant Unit, Cardiology Department of University Hospital 12 de Octubre, Facultad de Medicina UCM, Madrid, Spain.

9 Heart Failure and Transplant Unit, Cardiology Department of Hospital Universitario Reina Sofia, Córdoba, Spain.

10 Cardiology Department, Hospital General Universitario Gregorio Marañón, Madrid, Spain.

11 Heart Failure and Transplant Unit, Cardiology Department of Hospital de Sant Pau, Barcelona, Spain.

12 Hospital Universitario de Bellvitge, L'Hospitalet del Llobregat, Barcelona, Spain.

13 Heart Failure and Transplant Unit, Cardiology Department of Hospital Universitario Virgen del Rocio, Sevilla, Spain.

14 Heart Failure and Transplant Unit, Cardiology Department of Hospital Clinic, Barcelona, Spain.

15 Heart Failure and Transplant Unit, Cardiology Department of Hospital Universitario Central de Asturias, Oviedo, Spain.

16 Cardiac Surgery Department of Clinica, Universidad de Navarra, Pamplona, Navarra, Spain.

17 Heart Failure and Transplant Unit, Cardiology Department of Hospital Universitario Miguel Servet, Zaragoza, Spain.

18 Heart Failure and Transplant Unit, Cardiology Department of Hospital Clínico Universitario de Valladolid, Valladolid, Spain.

19 Heart Failure and Transplant Unit, Cardiology Department of Hospital Universitario Virgen de la Arrixaca, Murcia, Spain.

20 Pediatric Heart Transplant Unit, Hospital Universitario La Paz, Madrid, Spain.

21 Pediatric Heart Transplant Unit, Hospital Universitario Gregorio Marañón, Madrid, Spain.

22 Pediatric Heart Transplant Unit, Hospital Universitario Vall D'Hebron, Barcelona, Spain.

23 Universidade da Coruna (UDC), Instituto Universitario de Ciencias de la Salud, A Coruña, Spain. 


\section{Summary}

Male patients are at increased risk for developing malignancy postheart transplantation (HT); however, real incidence and prognosis in both genders remain unknown. The aim of this study was to assess differences in incidence and mortality related to malignancy between genders in a large cohort of HT patients. Incidence and mortality rates were calculated for all tumors, skin cancers (SCs), lymphoma, and nonskin solid cancers (NSSCs) as well as survival since first diagnosis of neoplasia. 5865 patients $(81.6 \%$ male) were included. Total incidence rates for all tumors, SCs, and NSSCs were lower in females [all tumors: 25.7 vs. 44.8 per 1000 person-years; rate ratio (RR) $0.68,(0.60-0.78), \mathrm{P}<0.001]$. Mortality rates were also lower in females for all tumors [94.0 (77.3-114.3) vs. $129.6(120.9-138.9)$ per 1000 person-years; RR 0.76, $(0.62-0.94), \mathrm{P}=0.01]$ and for NSSCs $[125.0(95.2-164.0)$ vs 234.7 (214.0-257.5) per 1000 person-years; RR 0.60 (0.44-0.80), $\mathrm{P}=0.001$ ], albeit not for SCs or lymphoma. Female sex was associated with a better survival after diagnosis of malignancy [log-rank p test $=0.0037$; HR 0.74 $(0.60-0.91), \mathrm{P}=0.004]$. In conclusion, incidence of malignancies post-HT is higher in males than in females, especially for SCs and NSSCs. Prognosis after cancer diagnosis is also worse in males.

\section{Key words}

Heart transplantation, malignancy, sexes

\section{Introduction}

Malignancy following heart transplantation (HT) is a main complication responsible for approximately $20 \%$ of deaths between 5 and 10 years following HT [1,2]. Despite improving survival in recent years, HT recipients have a greater risk of being diagnosed with cancer compared to the general population [3-5], partially related to chronic immunosuppression therapy [6], with a recent increasing trend, especially for skin cancer (SC) [7]. Apart from the International Society for Heart and Lung Transplantation (ISHLT) and the Spanish Post-HT Tumor (SPHTTR) registries, there have not been large studies evaluating the incidence, prognosis, and risk factors of malignancy development in HT survivors. The ISHLT registry recently reported a cumulative incidence of cancer post-transplant in survivors of $16.0 \%$ and of $27.9 \%$ at 5 and 10 years, respectively, mostly SCs [1]. On the other hand, the SPHTTR revealed an incidence rate of 25 and 34 per 1000 person-year at 5 and 10 years, respectively [8]. Furthermore, survival decreases in HT recipients following the diagnosis of de novo malignancy when compared with HT recipients without cancer [7].

Female recipients have a lower incidence of cancer than males [8, 9]. Some studies have reported male sex as an independent risk factor in multivariate analyses for developing malignancy [10], even excluding gender-specific tumors [11]. Nevertheless, there are no large studies informing the real incidence of malignancy for both sexes and moreover, it is unknown if the relative weight of death related to cancer following HT differs between genders. The SPHTTR is an online registry linked to the Spanish registry of HT that includes updated data on tumors of HT recipients provided by all centers performing HT in Spain since its initiation in this country in 1984 (19 centers; 16 adults and 3 pediatric centers). The aim of the present study, therefore, was to investigate differences in incidence and prognosis of malignancy between genders, using SPHTTR data to assess the requirement for gender-specific recommendations for HT patients.. 


\section{Patients and methods}

\section{Study population}

A retrospective cohort study of patients who underwent HT in Spanish centers between 1984 and June 2017 was performed. Data were extracted from the SPHTTR; information coming from centers with no current heart transplant program was excluded from the analysis. Adult patients (aged urn:xwiley:09340874:media:tri13827:tri13827-math-0001 16 years old) with follow-up or survival longer than 3 months following HT were included. However, re-transplant patients and those with combined organ transplantations were excluded. Patient data were recorded online until death or to December 2017 (follow-up termination), whichever occurred first.

\section{Variables}

Patients were allocated into two groups according to sex. Incidence rates (and 95\% confidence intervals (CIs)) of any cancer per 1000 person-year of follow-up following HT were calculated for each sex at 1,5 , and 10 years post-HT, as well as the corresponding rate ratio (RR) adjusted by age between genders. All analyses were also performed for SCs, lymphoma, nonskin solid cancers (NSSCs), and separately for the most frequent nongender-specific NSSCs (lung, gastrointestinal, and liver cancer). All types of SCs, including melanoma, were incorporated in the SC variable. Prostate, testicular, and on the other hand, breast, and gynecological cancers (endometrium, myometrium, ovary, fallopian tube, cervix, vulva, and vagina) were identified as male and female-specific NSSCs, respectively. Incidence rates for NSSCs and the corresponding rate ratio adjusted by age between genders were computed, both including and excluding these types of gender-specific malignancies separately, in order to assess their potential weight as confounders in the estimation of the risk of malignancy between genders.

All causes of mortality and the relative weight of cancer as cause of death were reported by gender and time following HT. Mortality rates of the different types of cancer per 1000 person-year and the corresponding RR adjusted by age between genders were calculated for both groups. Regarding NSSCs, mortality rates and female to male rate ratios were also estimated including and excluding gender-specific malignancies. Survival differences between sexes were calculated since the first diagnosis of cancer (any tumor).

\section{Statistical analysis}

Continuous variables were summarized as mean \pm standard deviation (SD) and compared using Student's $t$ tests or Mann-Whitney rank-sum tests depending on normality. Categorical variables were described as percentages and compared using chi-square or Fisher's exact tests accordingly. Incidence rates per 1000 person-year and their 95\% confidence intervals (CI) were calculated at 1, 5, and 10 years using the quadratic approximation to the Poisson log likelihood for the log-rate parameter. Female to male incidence and mortality rate ratios and their $95 \%$ CI were calculated using total incidence and mortality rates of both groups adjusted by age, respectively. Adjusted relative rate by age was calculated with Poisson regression models. On the other hand, cumulative incidence of all tumors, skin cancer, nonskin solid cancers, and lymphoproliferative disorders was estimated by means of a competing risk analysis, considering death as competing event. (The only competing event considered in this analysis was death). The cumulative incidence function was calculated using the model introduced/defined by Kalbfleisch and Prentice and the curves between genders were compared using Gray statistical test. The survival curves since first diagnosis of cancer were constructed by the Kaplan-Meier method using on the basis of all available follow-up data and were compared using the log-rank test to estimate the statistical differences between genders. A univariate Cox regression model to assess the influence of female sex in survival since first diagnosis of cancer was also performed. Statistical analyses were performed using STATA software version 12.0. The criterion for statistical significance was $P<0.05$. 


\section{Results}

\section{Study population}

A total of 7995 patients who underwent HT in Spain between 1984 and June 2017 were identified in the SPHTTR. 2130 patients were excluded from the analysis (441 pediatric patients, 157 combined transplantations, 174 re-transplant patients, 1292 with survival less than 3 months, and 45 with less than 3 months follow-up). Twenty one patients were also excluded because they were transplanted in a center with no current active HT program. Finally, 5865 adult HT patients were included in the analysis, 4788 males $(81.6 \%)$ and 1077 females $(18.4 \%)$.

Baseline characteristics of the cohort are displayed in Table 1 . Ischemic heart disease was the most common cause of heart failure in males pre-HT, but not in females (38\% vs. 15.3\%, respectively, $P<0.001)$. A higher prevalence of renal dysfunction and diabetes was observed in male recipients. Regarding the smoking status before HT, there were almost twice as many male than female smokers, with significant differences $(23.7 \%$ vs. $13.7 \%, P<0.001)$. In addition, smoking status after HT was also higher in male recipients $(4.4 \%$ vs. $1.9 \%, P<0.001)$. A history of malignancy pre-HT was more prevalent in female recipients $(7 \%$ vs. $2.5 \% P<0.001)$. No significant differences in the number of allograft rejections or in the prevalence of cytomegalovirus infection were found. In addition, there was a disparity between genders in initial immunosuppression therapy following HT, but no significant differences were identified in the use of induction treatment.

Table 1. Sociodemographic and clinical characteristics of heart transplantation (HT) recipients included in the analyses.

\begin{tabular}{|c|c|c|c|c|}
\hline Variable & Total & Male & Female & P-value \\
\hline $\mathrm{N}(\%)$ & $5865(100 \%)$ & $4788(81.6 \%)$ & $1077(18.4 \%)$ & \\
\hline Age & $52.0 \pm 11,5$ & $52.4 \pm 11.3$ & $50.2 \pm 12.4$ & $<0.001$ \\
\hline BMI & $25.4 \pm 3.2$ & $25.6 \pm 3.8$ & $24.4 \pm 4.5$ & $<0.001$ \\
\hline \multicolumn{5}{|l|}{ Etiology } \\
\hline Ischemic heart disease & 33.9 & 38.0 & 15.3 & $<0.001$ \\
\hline IDC & 29.8 & 29.2 & 32.5 & \\
\hline Other & 28.6 & 25.6 & 41.7 & \\
\hline Renal dysfunction & 15.7 & 16.9 & 10.5 & $<0.001$ \\
\hline Diabetes & 15.4 & 16.2 & 12.0 & 0.001 \\
\hline Smoker pre-HT & 21.8 & 23.7 & 13.7 & $<0.001$ \\
\hline Smoker post-HT & 3.8 & 4.4 & 1.9 & $<0.001$ \\
\hline Neoplasia pre-HT & 3.3 & 2.5 & 7.0 & $<0.001$ \\
\hline Antiviral prophylaxis post-HT & 37.0 & 36.7 & 38.5 & 0.288 \\
\hline Asymptomatic CMV infection & 19.5 & 19.0 & 21.6 & 0.095 \\
\hline Symptomatic CMV infection & 10.5 & 10.2 & 11.8 & 0.189 \\
\hline Allograft rejection (number) & $1.1 \pm 1.5$ & $1.1 \pm 1.4$ & $1.2 \pm 1.6$ & 0.800 \\
\hline Induction therapy $(\%)$ & 83.0 & 82.6 & 84.8 & 0.087 \\
\hline \multicolumn{5}{|l|}{ Initial treatment } \\
\hline Cyclosporine & 64.9 & 68.3 & 49.9 & $<0.001$ \\
\hline Tacrolimus & 33.3 & 29.8 & 48.9 & $<0.001$ \\
\hline Azathioprine & 39.2 & 40.4 & 33.5 & $<0.001$ \\
\hline Everolimus/Sirolimus & 2.8 & 2.8 & 2.9 & 0.942 \\
\hline MMF & 58.4 & 57.1 & 64.1 & $<0.001$ \\
\hline Steroids & 98.6 & 98.6 & 98.3 & 0.466 \\
\hline
\end{tabular}

BMI, Body Mass Index; CMV, cytomegalovirus; HCV, hepatitis C virus; IDC, idiopathic dilated cardiomyopathy; MMF, mycophenolate mofetil. 
During follow-up (median follow-up 103 months), 1473 patients [1274 males (26.6\%) and 199 females (18.5\%)] developed a total of 2278 malignancies [2015 (88.45\%) and $263(11.55 \%)$ between males and females, respectively]. A total of $1.93 \pm 2.15$ tumors per patient were described in the cohort $(2$ \pm 2.25 in males and $1.38 \pm 0.82$ in females, $\mathrm{P}<0.001$ ). The most frequently diagnosed cancer was $\mathrm{SC}$, representing $54.2 \%$ of all tumors, followed by NSSCs $(39.9 \%)$, with lung cancer being the most frequent of this group of tumors ( $11.4 \%$ of all cancers). Lymphoproliferative disorders were less common $(5.9 \%$ of all tumors). In gender-specific malignancies, there were 136 male (132 prostate, 4 testicular) and 51 female tumors ( 27 gynecological, 24 breast cancer). No breast cancer was reported in male recipients.

On the other hand, a disparity in incidence rates between genders was found. Female recipients had a total malignancy incidence rate of 25.7 per 1000 person-year, significantly lower than the male incidence rate of 44.8 (RR $0.6895 \%$ CI $0.60-0.78, \mathrm{P}<0.001$ ). In both genders the most common malignancy was $\mathrm{SC}$, but females had lower risk than males with a total incidence rate of 12.6 and 24.4 per 1000 personyear, respectively (RR $0.6295 \%$ CI $0.52-0.74, \mathrm{P}<0.001$ ).

Lymphoproliferative disorders were less incident than SCs (5.92\% of all tumors) and no significant differences were found in total incidence rates between genders. In addition, there was no increasing trend in incidence for lymphoproliferative disorders over time, which differed from those observed for SCs and de novo NSSCs. The total incidence rate for lymphoma was 2.0 and 2.5 per 1000 person-year for female and male recipients, respectively (RR $0.8495 \%$ CI $0.52-1.36, \mathrm{P}=0.483$ ).

The incidence rate of de novo NSSCs was lower in females than males (11.1 vs. 17.5 per 1000 personyear; RR $0.7595 \%$ CI $0.62-0.92, \mathrm{P}=0.005)$. This result was predominantly attributable to the lower incidence of lung cancer in female recipients (1.1 vs. 5.5 per 1000 person-year; RR $0.2395 \%$ CI $0.13-$ $0.43, \mathrm{P}<0.001)$. These differences in the incidence rate of the novo NSSCs remained statistically significant when gender-specific malignancies were excluded (6.4 vs. 14.5 per 1000 person-year; RR 0.52 $95 \%$ CI $0.40-0.67, \mathrm{P}<0.001)$. No disparity was found between genders when analyzing liver cancer $(2.3$ vs. 3.1 per 1000 person-year; RR $0.6695 \%$ CI $0.23-1.86, \mathrm{P}=0.428)$ or gastrointestinal cancer $(0.4$ vs 0.7 per 1000 person-year; RR $0.8595 \%$ CI $0.55-1.32, \mathrm{P}=0.474$ ) separately (Table 2 ).

Cumulative incidence considering competitive risk of death was also higher in male recipients than females for all tumors $(\mathrm{P}<0.001)$, SCs $(\mathrm{P}<0.001)$, NSSCs $(\mathrm{P}=0.002)$, and lung cancer $(\mathrm{P}<0.001)($ Fig. 1). Nevertheless, these disparities in cumulative incidence between genders were not observed when analyzing lymphoproliferative disorders $(\mathrm{P}=0.728)$ and gastrointestinal $(\mathrm{P}=0.509)$ and liver cancers $(\mathrm{P}$ $=0.415)$. Because of the greater prevalence of smoking history in males and because of the wellestablished relationship between tobacco use and cancer risk, a sub-analysis was performed to assess the weight of smoking as a mediator of the previously analyzed results. After adjusting the total incidence female to male rate ratios by age and smoking status, similar outcomes were found. In this sub-analysis, female recipients maintained a lower risk of developing any tumor (25.7 vs. 44.8 per 1000 person-year, RR 0.64 CI 95\% 0.55-0.75; P < 0.001). The only difference in the results of this sub-analysis was in the risk of developing NSSCs, which remained higher in the male group, but lost statistical significance (11.1 vs. 17.5 per 1000 person-year, RR 0.80 CI 95\% 0.63-1.02; P=0.069). In the sub-analysis, males also had higher incidence of SCs (12.6 vs. 24.4 per 1000 person-year, RR 0.52 CI 95\% $0.41-0.65 \mathrm{P}<0.001)$ and NSSCs when excluding gender-specific malignancies (6.4 vs. 14.5 per 1000 person-year, RR 062 CI 95\% $0.46-0.83 \mathrm{P}=0.001)$. The greater prevalence of smoking status in males did not change the higher risk for developing lung cancer in male recipients (1.1 vs. 5.5 per 1000 person-year, RR 0.37 CI 95\% 0.19 $0.69 ; \mathrm{P}<0.001)$ (Table S1).

\section{Mortality and prognosis of malignancy}

Mortality following HT, causes of mortality, and the relative weight of deaths caused by malignancy at different time periods following HT were analyzed in both genders (Fig. 2). At follow-up completion, 2780 deaths were registered and $584(21.0 \%)$ were due to causes related to malignancies. Differences in cancer as a cause of death were observed between genders. Malignancy was the cause of death in $22.4 \%$ of males and $12.5 \%$ of females $(\mathrm{P}<0.001)$; this difference reached statistical significance at 5 years following HT (Table S2). 
Like incidence, mortality rates for all tumors were significantly lower in female recipients than in male recipients (94.0 vs. 129.6 per 1000 person-year, RR $0.76,95 \%$ CI $0.62-0.94, \mathrm{P}=0.01$ ). This disparity in the relative weight of cancer as cause of death was mainly due to a lower mortality rate of de novo NSSCs observed in female recipients (125.0 vs. 234.7 per 1000 person-year, RR $0.6095 \%$ CI 0.44 $0.80, \mathrm{P}=0.001$ ), although this finding was not statistically significant for lung cancer, liver cancer or gastrointestinal cancer analyzed separately (Table 3). Nevertheless, when analyzing mortality rates of NSSCs excluding gender-specific malignancies, this disparity remained statistically significant (RR 0.52 95\% CI $0.36-0.74, \mathrm{P}=0.001)$. No differences were observed between genders in mortality rates due to cutaneous cancers or to lymphoproliferative disorders. However, female sex was associated with a better survival since the first diagnosis of malignancy when compared to male recipients (log-rank $\mathrm{P}$ test $=$ 0.0037; HR 0.74 (0.60-0.91), $\mathrm{P}=0.004$ ) (Fig. 3).

Table 2. Incidence rate of all cancers, skin cancer, lymphoma, and nonskin solid cancers (including and excluding gender-specific malignancies) at 1,5 , and 10 years of follow-up by gender and total cumulative incidence female to male rate ratios of the different types of malignancy adjusted by age in heart transplantation recipients.

\begin{tabular}{|c|c|c|c|c|c|c|c|}
\hline \multirow[t]{2}{*}{ Type of tumor } & \multirow[t]{2}{*}{ Time } & \multicolumn{2}{|l|}{ Male } & \multicolumn{2}{|l|}{ Female } & \multicolumn{2}{|c|}{$\begin{array}{l}\text { Female to Male } \\
\text { Incidence RR }\end{array}$} \\
\hline & & $\begin{array}{l}\text { Person-year } \\
\text { (tumors) }\end{array}$ & $\begin{array}{l}\text { Incidence rate } \\
(95 \% \mathrm{CI})(*)\end{array}$ & $\begin{array}{l}\text { Person-year } \\
\text { (tumors) }\end{array}$ & $\begin{array}{l}\text { Incidence rate } \\
(95 \% \mathrm{CI})(*)(-)\end{array}$ & RR & P-value \\
\hline \multirow[t]{4}{*}{ All tumors } & 1 year & $4631.4(87)$ & $18.8(15.2-23.2)$ & $1046.2(14)$ & $13.4(7.9-22.6)$ & & \\
\hline & 5 year & $\begin{array}{l}19677.8 \\
(538)\end{array}$ & $27.3(25.1-29.8)$ & $4464.8(77)$ & $17.3(13.8-21.6)$ & & \\
\hline & 10 year & $\begin{array}{l}32796.2 \\
(1255)\end{array}$ & $38.3(36.2-40.4)$ & $7398.3(155)$ & $21.0(17.9-24.5)$ & & \\
\hline & Total & $\begin{array}{l}45004.1 \\
(2015)\end{array}$ & $44.8(42.9-46.8)$ & $\begin{array}{l}10239.0 \\
(263)\end{array}$ & $25.7(22.8-29.0)$ & $\begin{array}{l}0.68 \\
(0.60-0.78)\end{array}$ & $<0.001$ \\
\hline \multirow[t]{4}{*}{ Skin } & 1 year & $4631.4(25)$ & $5.4(3.7-8.0)$ & $1046.2(3)$ & $2.9(0.9-8.9)$ & & \\
\hline & 5 year & $\begin{array}{l}19677.8 \\
(227)\end{array}$ & $11.5(10.1-13.1)$ & $4464.8(28)$ & $6.3(4.3-9.1)$ & & \\
\hline & 10 year & $\begin{array}{l}32796.2 \\
(648)\end{array}$ & $19.8(18.3-21.3)$ & 73983 (69) & $9.3(7.4-11.8)$ & & \\
\hline & Total & $\begin{array}{l}45004.1 \\
(1099)\end{array}$ & $24.4(23.0-25.9)$ & $\begin{array}{l}10239.0 \\
(129)\end{array}$ & $12.6(10.6-15.0)$ & $\begin{array}{l}0.62 \\
(0.52-0.74)\end{array}$ & $<0.001$ \\
\hline \multirow[t]{4}{*}{ Lymphoma } & 1 year & $\begin{array}{l}4631.4 \\
(16)\end{array}$ & $3.5(2.1-5.6)$ & $1046.2(3)$ & $2.9(0.9-8.9)$ & & \\
\hline & 5 year & $\begin{array}{l}19677.8 \\
(46)\end{array}$ & $2.3(1.8-3.1)$ & $4464.8(8)$ & $1.8(0.9-3.6)$ & & \\
\hline & 10 year & $\begin{array}{l}32796.2 \\
(79)\end{array}$ & $2.4(1.9-3.0)$ & $7398.3(13)$ & $1.8(1.0-3.0)$ & & \\
\hline & Total & $\begin{array}{l}45004.1 \\
(114)\end{array}$ & $2.5(2.1-3.0)$ & $10239.0(20)$ & $2.0(1.3-3.0)$ & $\begin{array}{l}0.84 \\
(0.52-1.36)\end{array}$ & 0.483 \\
\hline \multirow[t]{4}{*}{$\begin{array}{l}\text { NNSC } \\
\text { (including GSN) }\end{array}$} & 1 year & $4631.4(46)$ & $9.9(7.4-13.3)$ & $1046.2(8)$ & $7.7(3.8-15.3)$ & & \\
\hline & 5 year & $\begin{array}{l}19677.8 \\
(262)\end{array}$ & $13.3(11.8-15.0)$ & $4464.8(41)$ & $9.2(6.8-12.5)$ & & \\
\hline & 10 year & $\begin{array}{l}32796.2 \\
(517)\end{array}$ & $15.8(14.5-17.2)$ & $7398.3(73)$ & $9.9(7.8-12.4)$ & & \\
\hline & Total & $\begin{array}{l}45004.1 \\
(789)\end{array}$ & $17.5(16.4-18.8)$ & $\begin{array}{l}10239.0 \\
(114)\end{array}$ & $11.1(9.3-13.4)$ & $\begin{array}{l}0.75 \\
(0.62-0.92)\end{array}$ & 0.005 \\
\hline $\begin{array}{l}\text { NSCC } \\
\text { (excluding GSN) }\end{array}$ & Total & $\begin{array}{l}45004.1 \\
(654)\end{array}$ & $14.5(13.5-15.7)$ & $10239.0(11)$ & $6.4(5.0-8.1)$ & $\begin{array}{l}0.52 \\
(0.40-0.67)\end{array}$ & $<0.001$ \\
\hline Lung cancer & Total & $\begin{array}{l}45004.1 \\
(248)\end{array}$ & $5.5(4.9-6.2)$ & $10239,0(11)$ & $1.1(0.6-1.9)$ & $\begin{array}{l}0.23 \\
(0.13-0.43)\end{array}$ & $<0.001$ \\
\hline GI cancer & Total & $\begin{array}{l}45004.1 \\
(141)\end{array}$ & $3.1(2.7-3.7)$ & $10239,0(23)$ & $2.3(1.5-3.4)$ & $\begin{array}{l}0.85 \\
(0.55-1.32)\end{array}$ & 0.474 \\
\hline Liver cancer & Total & $\begin{array}{l}45004.1 \\
(32)\end{array}$ & $0.7(0.5-1.0)$ & $10239,0(4)$ & $0.4(0.2-1.0)$ & $\begin{array}{l}0.66 \\
(0.23-1.86)\end{array}$ & 0.428 \\
\hline
\end{tabular}

CI, confidence interval; GI, gastrointestinal cancer; GSN, gender-specific neoplasm; NSSC, nonskin solid cancer; RR, rate ratio.

* Per 1000 person-year. 

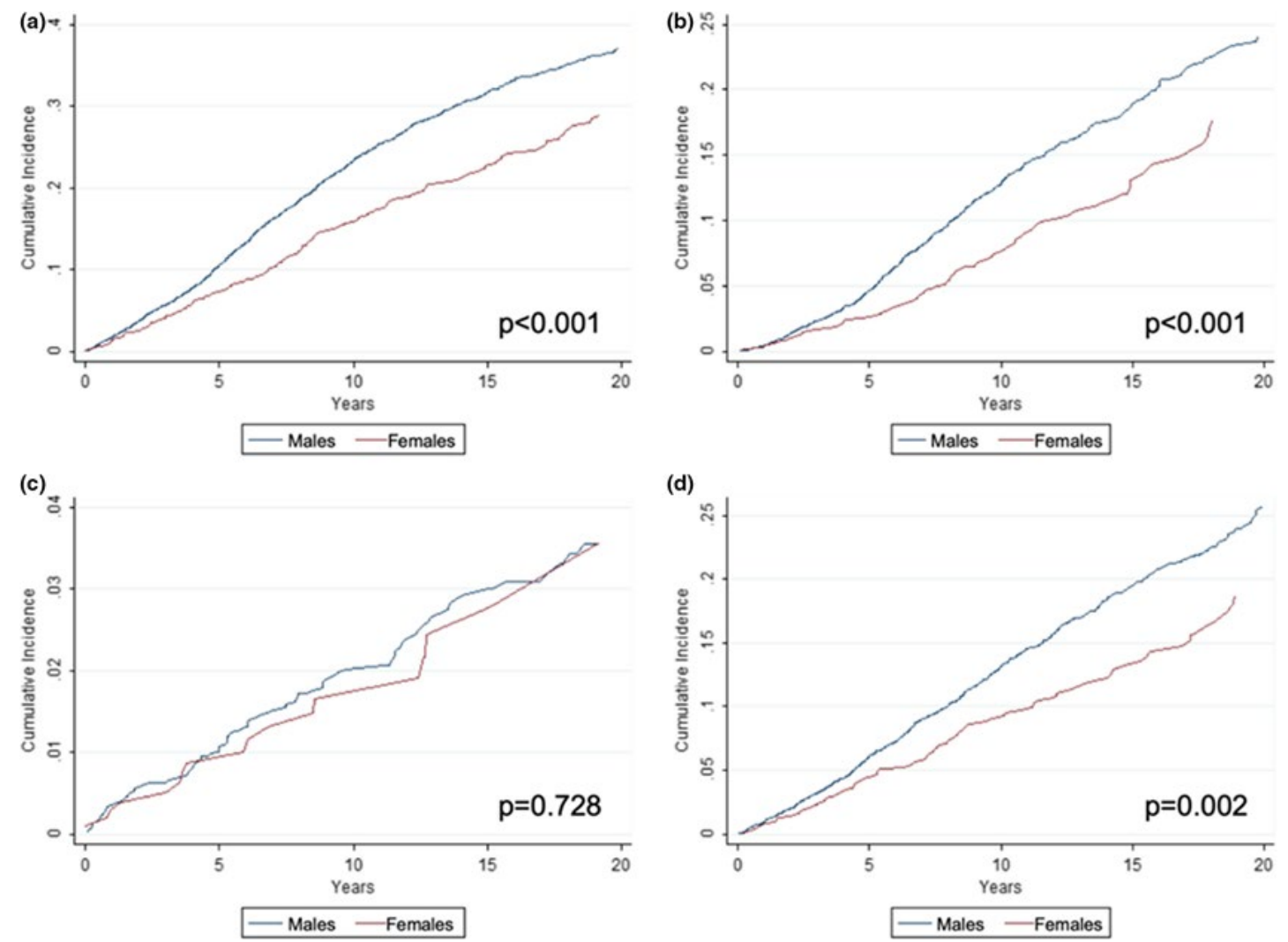

Figure 1. Cumulative incidence considering competitive risk of death in heart transplantation (HT) recipients for all tumors (a); skin cancers (b); lymphoma (c); and nonskin solid cancers (d). Cumulative incidence considering competitive risks of death was significantly higher in males than females for all tumors, SCs, NSSCs, and lung cancer, but not for lymphoma. RR, rate ratio.

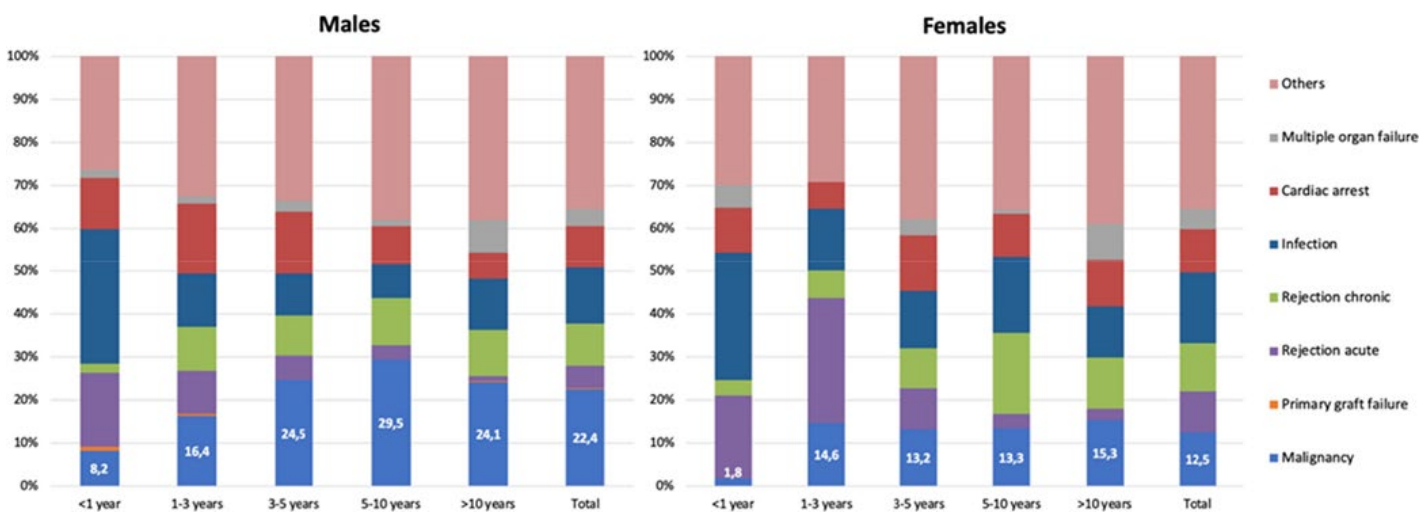

Figure 2. Distribution of different causes of death between genders for different time periods following heart transplantation (HT). Representation of the percentage of cancers as causes of death in both genders at different time periods following HT. The difference in mortality related to cancer in both sexes reached statistical significance at 5 years following HT. 
Table 3. Mortality rates and female to male mortality rate ratios adjusted by age between genders and by different types of tumors in heart transplantation recipients.

\begin{tabular}{|c|c|c|c|c|c|c|}
\hline \multirow[t]{2}{*}{ Type of tumor } & \multicolumn{2}{|l|}{ Female } & \multicolumn{2}{|l|}{ Male } & \multicolumn{2}{|c|}{ Female to male mortality RR } \\
\hline & Mortality rate $(*)$ & CI $95 \%$ & Mortality rate $(*)$ & CI 95\% & $\mathrm{RR}$ & P-value \\
\hline All tumors & 94.0 & $77.3-114.3$ & 129.6 & $120.9-138.9$ & $0.76(0.62-0.94)$ & 0.01 \\
\hline Skin cancer & 63.2 & $45.4-88.0$ & 70.4 & $62.6-79.1$ & $0.88(0.62-1.25)$ & 0.481 \\
\hline Lymphoma & 137.8 & $80.0-237.3$ & 237.5 & $187.9-300.2$ & $0.58(0.32-1.06)$ & 0.076 \\
\hline $\begin{array}{l}\text { NSSC } \\
\text { (including GSN) }\end{array}$ & 125.0 & $95.2-164.0$ & 234.7 & $214.0-257.5$ & $0.60(0.44-0.80)$ & 0.001 \\
\hline $\begin{array}{l}\text { NSSC } \\
\text { (excluding GSN) }\end{array}$ & & & & & $0.52(0.36-0.74)$ & $<0.001$ \\
\hline Lung cancer & 248.6 & $103.5-597.4$ & 575.9 & $494.8-670.2$ & $0.47(0.19-1.14)$ & 0.095 \\
\hline $\begin{array}{l}\text { Gastrointestinal } \\
\text { cancer }\end{array}$ & 123.0 & $69.9-216.6$ & 188.7 & $151.2-235.6$ & $0.60(0.33-1.10)$ & 0.100 \\
\hline Liver cancer & 201.7 & $28.4-1431.8$ & 746.0 & $500.1-1113.1$ & $0.16(0.02-1.20)$ & 0.074 \\
\hline
\end{tabular}

CI, confidence interval; GSN, gender-specific malignancy; NSSC, nonskin solid cancer; RR, rate ratio.

* Per 1000 person-year.

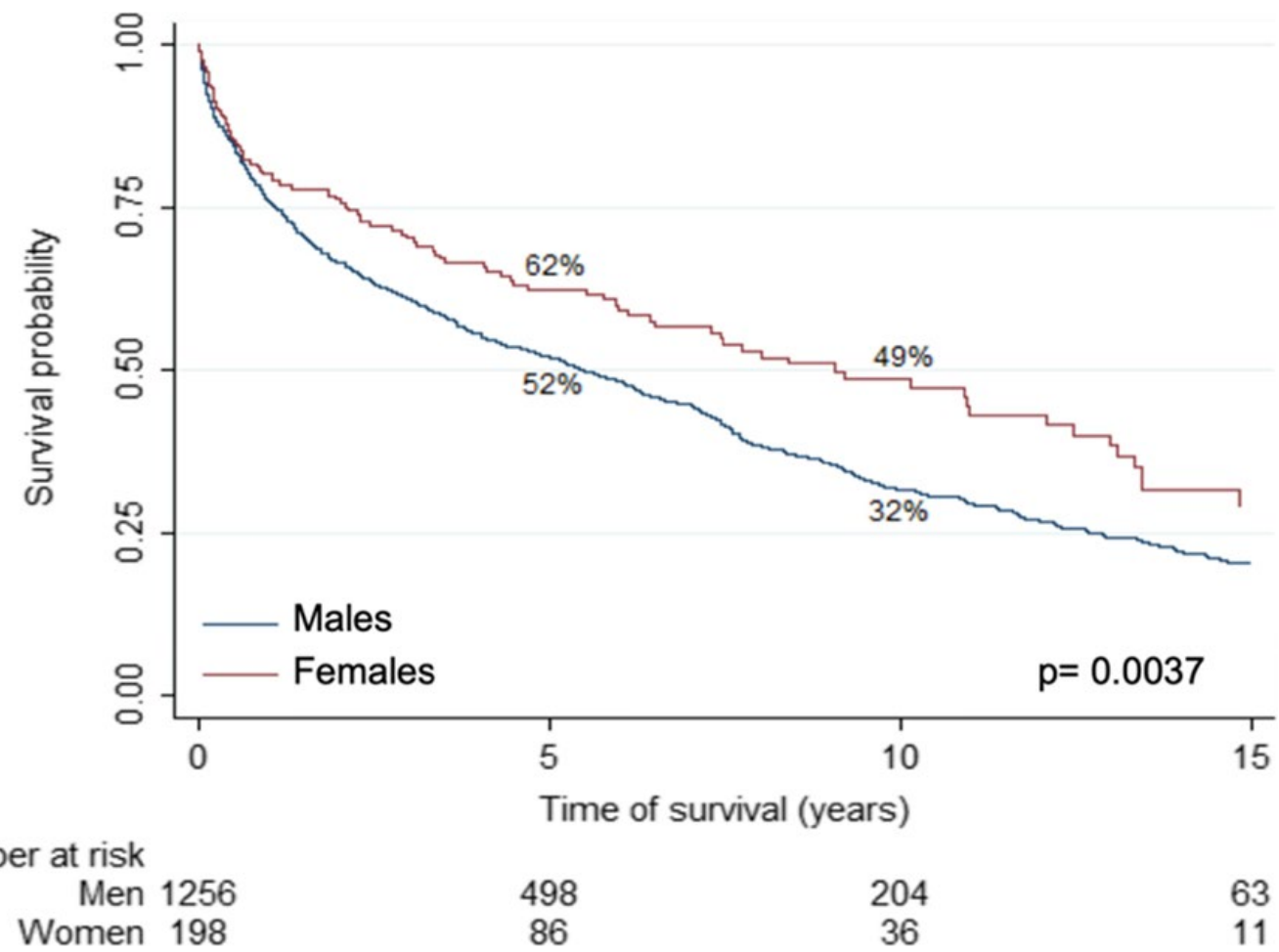

Figure 3. Survival of heart transplantation recipients since first diagnosis of malignancy in both genders. Survival since first diagnosis of malignancy is lower in males. 


\section{Discussion}

To the best of our knowledge, this is the first large study assessing differences between genders in the incidence and the prognosis of malignancy in post-HT patients. The main findings of our research were the following: (1) the incidence of malignancies post-HT is higher in males than in females, both in total cancers and according to the type of tumor, except for lymphoma, liver, and gastrointestinal cancers; (2) the relative weight of cancer as cause of death tends to be higher among males and reaches conventional statistical significance after 5 years; and (3) the difference in incidence of malignancy is not due to gender-specific malignancies or tobacco abuse.

Assessing post-HT malignancy incidence and prognosis could be challenging because it is a long-term complication that could even occur more than once in the same recipient, especially cutaneous cancers. Moreover, the attribution of cancer as cause of death in registries may be hidden by other complications arising in the management of malignancies (e.g., rejection as a consequence of reducing immunosuppression or infection) that are equally fatal. Furthermore, most registries report only frequency data and lack of temporal trend analysis.

Malignancy, a well-known late complication following solid organ transplantation, has been reported by previous studies to have a higher incidence in males than in females[12]. However, no specific analysis including both genders has been performed specifically in HT patients. In our large cohort study, incidence rates of all tumors, SCs, and de novo NSSCs post-HT were also higher in males. In fact, there was an increasing trend during follow-up in both genders for these malignancies, although not for lymphoma, whose incidence remained unchanged over time, as previously described in another analysis of the same cohort [9].

In this registry and in corroborating previous studies, $\mathrm{SC}$ was the most frequent malignancy diagnosed following HT[7]. Previous studies reported that Caucasian solid organ transplant recipients were at higher risk for SCs than non-Caucasians[13]. It is important to note that Spain is a country with a predominantly Caucasian population and high sunlight exposure, which is another factor related to the develop of SCs in this group of patients[14]. This could also explain the higher incidence observed for SCs in our study. Youn et al., using data from 17,587 HT recipients from the ISHTL registry, recently reported a cumulative incidence of $8.4 \%$ SCs within 5 years following HT during 2006-2011, significantly greater than that reported in the previous period (2000-2005)[7]. In their research, they also identified male sex as a risk factor for developing SCs in a multivariate analysis (female to male HR: $0.61(0.48-0.78), \mathrm{P}<$ 0.0001). Asleh et al., assessing differences in the incidence of malignancy following HT depending on whether a calcineurin inhibitor or sirolimus was used in a smaller cohort, reported an incident rate of nonmelanoma SCs of 5.3 per 100 person-year; however, in this case, male sex was only identified as a risk factor in a univariate, but not in a multivariate, analysis (HR $1.18(0.82-1.70), \mathrm{P}=0.374)$ [15]. Despite the fact that SC is a malignancy with an increasing incidence in the general population[16], the risk of its development is even greater in recipients of solid organ transplantation[17]. In our cohort, SCs also had an incidence rate significantly lower in females (RR $0.6295 \% \mathrm{CI} 0.52-0.74, \mathrm{P}<0.001$ ). It is important to highlight that this difference in the incidence of SC between genders is not only observed in HT patients, but also in other solid organ transplants recipients on immunosuppression therapy[18].

In our study, the incidence of lung cancer was strongly associated with male sex (RR 0.23 CI 95\% 0.13-0.43, $\mathrm{P}<0.001)$. This finding is comparable with that in the general population in which the incidence of lung cancer remains higher in males, despite an inverse trend between genders in the last decade (mainly in high socioeconomic status countries) that is explained by a decline in male smoking prevalence during this period[19]. This association could possibly be related to the prevalent smoking history pre-HT in males. However, in our study cohort, the incidence of lung cancer remained higher in males after adjusting the results by tobacco use. 
The reason for the disparity in incidence of malignancy between sexes remains unknown, although the same trend is also observed in the general population[20]. One possible explanation could be gender differences in pharmacokinetics of immunosuppression therapy. Weight-normalized clearance of calcineurin inhibitors and sirolimus is higher in females than in males[21]. This could lead to a more intensive immunosuppression regimen in male recipients, and consequently, to a higher risk for developing malignancy. However, this argument is not consistent with the fact that, in contrast, female sex is associated with a lower clearance of mycophenolic acid and prednisolone[22, 23], and because close drug monitoring to achieve therapeutic doses is routinely performed without sex-specific recommendations[24]. On the other hand, in our study there were significant differences in baseline immunosuppression therapy which could be a reasonable explanation for the differences in incidence of cancer between genders. Males had a higher baseline use of azathioprine and cyclosporine and less use of tacrolimus and mycophenolic acid than females in our cohort. Cyclosporine stimulates carcinogenesis possibly mediated by transforming growth factor-beta 1 which enhances survival, progression, and metastasis of established tumors and inhibits DNA repair [25]. In addition, Tacrolimus has a lower incidence of de novo solid tumors compared with cyclosporine [26] and on the other hand, the use of mycophenolic acid may be more protective against malignancy [9, 27]. However, the SPHTTR did not register changes in immunosuppression therapy throughout follow-up. This could explain the higher use of azathioprine and cyclosporine in the cohort at baseline and the lower use of mammalian target-ofrapamycin inhibitors which have been also associated with a lower incidence of malignancy after HT [15].

Another possible reason could be the significantly more frequent smoking history pre-HT in males observed in the study. Even though smoking is intensely associated with lung cancer, it is also related to other NSSCs, including bladder, kidney, pharynx, liver, pancreas, and stomach[28]. In our registry, 596 of the NSSCs diagnosed, including lung cancer (66\% of all NSSCs and $26.1 \%$ of all tumors), were malignancies that have been associated with tobacco use in the general population. This could explain that, when adjusting the results by age and smoking history, there was a higher risk of developing NSSCs in males, which was not significant. However, the effect of tobacco abuse in the risk of developing a NSSC was relatively small, and significant differences were also observed between genders when excluding sex-specific malignancies. In this regard, in a recent study, other cardiovascular risk factors, in addition to sex and tobacco, such as age and arterial hypertension, were independently related to an increased risk of developing cancer in a large cohort of 12712 patients[29]. On the other hand, the relative weight of gender-specific malignancies that could have been the reason for this discrepancy in incidence and mortality in NSSCs did not change the results when they were excluded from the analysis, probably due to the low number reported ( $8.21 \%$ of all tumors).

Together with cardiac allograft vasculopathy, malignancy is, in fact, one of the leading causes of death in the long-term follow-up of HT patients[1, 2]. Transplant outcomes have improved dramatically over time; however, the increased age of recipients and consequent longer immunosuppressive therapy administered to prevent graft rejection are associated with an additional risk of developing cancer[9]. Survival is markedly decreased in solid organ transplant recipients after de novo malignancy when compared with patients without malignancy[7] and when compared with cancer patients in the general population[30]. However, no differences between genders have been previously reported in HT patients, in contrast to nonimmunosuppressed people in which cancer survival tends to be lower in males[31, 32]. In our cohort, survival rates were lower for males than females following diagnosis of malignancy. This difference could be a consequence of the underlying higher incidence of malignancy in males, specifically of NSSCs with worse outcomes, such as lung cancer. 
Even though treatment of malignancies is important, prevention is the essential key for improving outcomes. ISHTL guidelines recommend close SC surveillance, annual dermatological examinations, and screening recommendations for common malignancies (breast, colon, and prostate) similar to those recommended for nonimmunosuppressed individuals. In addition, immunosuppressive treatment should be minimized as much as possible, particularly in patients at high risk for malignancy[24]. However, there are no current gender-specific recommendations for immunosuppressed post-transplant patients. In our registry, male recipients had significantly higher incidence of SC, although no impact prognosis in terms of mortality was observed when compared to females. The small differences in prognosis in SCs may not warrant the need for sex-specific recommendations besides those that are already suggested. In fact, only de novo NSSCs were associated with an increased mortality rate in male recipients in this study. However, this analysis included a wide range of malignancies, and when separately analyzed, no significant differences in terms of mortality were found in both genders for lung, gastrointestinal or liver cancers. Moreover, controversies exist about cancer screening in this population because of reduced life expectancy and competing causes of death[33].

This study has some limitations, mainly due to its retrospective design. First of all, we were not able to verify the detailed underlying mechanisms for the increased incidence of malignancy in male recipients. Regarding smoking status before HT, when a patient ceased smoking or how long he/she was exposed was not registered. In addition, both groups differ in some basal characteristics; however, the results were adjusted by age and tobacco which are the most important risk factors for the development of cancer. Although initial immunosuppression therapy treatment differed between both genders, the SPHTTR did not register changes throughout follow-up. In this regard, immunosuppressive therapies have changed along the last decades, which could explain the higher use of Azathioprine and Cyclosporine in both groups. Although providing era-specific malignancy rates stratified by gender could have been interesting, we would not have been able to specify if the differences observed were due to differences in immunosuppression therapy or to other change in the therapeutic management. In order to correct this bias, all the incidence and mortality rates were adjusted by age, the most important risk factor for cancer. Finally, although higher incidence and mortality rates were found for NSSCs in males than in females, because of the multiple types of tumors included, we could not identify which kinds of malignancies were responsible for this disparity.

In conclusion, the incidence of malignancy following HT is higher in males, specifically due to SCs and NSSCs, but not for lymphoma. Prognosis after a cancer diagnosis is worse in males. The relative weight of death due to malignancies is higher in males, and after 5 years these differences reach statistical significance, mostly due to NSSCs. However, these results are not explained by the disparity in the incidence of gender-specific malignancies or by the higher prevalence of smokers in male recipients.

\section{Authorship}

CM: analyzed data and wrote the paper. MG, LA, EB, FG, JFD, JMAP, IS, SM, JG, JMS, FP, BD, GRJ, TB, LF, IG, LG, MC, and DCA: reviewed manuscript. JM: designed study and collected data. MGC: designed study, analyzed data, and wrote the paper.

\section{Funding}

This work has been co-financed with FEDER funds (Instituto de Salud Carlos III).

\section{Conflict of interest}

The authors of this manuscript have no conflicts of interest to disclose as described by Transplant International. 


\section{Acknowledgements}

The authors acknowledge the contributions of the following investigators who also participated in the Spanish Post-heart Transplant Tumour Registry: Gonzalo Barge-Caballero, Paula Blanco-Canosa, David Couto-Mallón, Zulaika Grille-Cancela, Maria J. Paniagua-Martin, Luis Alonso-Pulpón Rivera, Francisco Hernández Pérez, Javier Segovia Cubero, Mónica Cebrián Pinar, Raquel López Vilella, Luis Martínez Dolz, Soledad Martínez Penades, Ignacio Sánchez Lázaro, Jose Antonio Vazquez de Prada, Maria Dolores García-Cosio, Laura Morán Fernández, Pedro Caravaca, Zorba Blázquez, Francisco Carrasco Ávalos, Amador Lopez Granados, Juan Fernández-Yáñez, Manuel Martínez-Sellés, Paula Navas Tejedor, Carlos Ortiz Bautista, María Jesús Valero Masa, Adolfo Villa Arranz, Eduardo Zatarain Nicolás, Vicens Brossa Loidi, Laura López, Eulalia Roig Minguell, Nicolás Manito Lorite, Josep Roca, Carles Díez López, Elena García Romero, Antonio Grande-Trillo, Diego Rangel-Sousa, José Luis Lambert Rodríguez, María José Bernardo Rodríguez, Ana Portoles Ocampo, Marisa Sanz Julve, Amada Recio Platero, Domingo Pascual Figal, Francisco Pastor Pérez, and Nuria Gil Villanueva.

\section{Supporting information}

Additional supporting information may be found online in the Supporting Information section at the end of the article. Table S1. Total incidence rate of all cancers, skin cancers, lymphoma and non-skin solid cancer (including and excluding gender-specific malignancies) and female to male rate ratios adjusted by age and smoking status in heart transplantation recipients. Table S2. Proportion of cancer deaths in male and female recipients by time period following heart transplantation (HT).

\section{References}

1. Khush KK, Cherikh WS, Chambers DC, et al. The International Thoracic Organ Transplant Registry of the International Society for Heart and Lung Transplantation: Thirty-fifth Adult Heart Transplantation Report-2018; Focus Theme: Multiorgan Transplantation. J Heart Lung Transplant. 2018; 37: 1155.

2. González-Vílchez F, Almenar-Bonet L, Crespo-Leiro MG, et al. Spanish Heart Transplant Registry. 29th Official Report of the Spanish Society of Cardiology Working Group on Heart Failure. Rev Española Cardiol (English Ed) 2018; 71: 952.

3. Higgins RS, Brown RN, Chang PP, et al. A multi-institutional study of malignancies after heart transplantation and a comparison with the general United States population. J Hear Lung Transplant 2014; 33: 478.

4. Crespo-Leiro MG, Villa-Arranz A, Manito-Lorite $\mathrm{N}$, et al. Lung cancer after heart transplantation: results from a large multicenter registry. Am J Transplant 2011; 11: 1035.

5. Na R, Grulich AE, Meagher NS, McCaughan GW, Keogh AM, Vajdic CM. Comparison of de novo cancer incidence in Australian Liver, heart and lung transplant recipients. Am J Transplant 2013; 13: 174 .

6. Dantal J, Soulillou J-P. Immunosuppressive drugs and the risk of cancer after organ transplantation. N Engl J Med 2005; 352: 1371.

7. Youn J-C, Stehlik J, Wilk AR, et al. Temporal trends of de novo malignancy development after heart transplantation. J Am Coll Cardiol 2018; 71: 40.

8. Crespo-Leiro MG, Alonso-Pulpón L, Delgado Jiménez JF, et al. I Informe del Registro Español de Tumores Postrasplante Cardiaco. Rev Española Cardiol Supl 2015; 15(Supl.B): 50 .

9. Crespo-Leiro MG, Alonso-Pulpón L, Vázquez de Prada JA, et al. Malignancy after heart transplantation: incidence, prognosis and risk factors. Am J Transplant 2008; 8: 1031.

10. Delgado JF, Alonso-Pulpón L, Mirabet S, et al. Cancer incidence in heart transplant recipients with previous neoplasia history. Am J Transplant 2016; 16: 1569.

11. Van Keer J, Droogné W, Van Cleemput J, et al. Cancer after heart transplantation: a 25-year single-center perspective. Transplant Proc 2016; 48: 2172.

12. Engels EA, Pfeiffer RM, Fraumeni JF, et al. Spectrum of cancer risk among US solid organ transplant recipients. JAMA 2011; 306: 1891.

13. Cohen BE, Krivitskiy I, Bui S, et al. Comparison of skin cancer incidence in Caucasian and Non-Caucasian liver vs. lung transplant recipients: a tale of two regimens. Clin Drug Investig 2019; 39: 197.

14. Molina BD, Leiro MGC, Pulpón LA, et al. Incidence and risk factors for nonmelanoma skin cancer after heart transplantation. Transplant Proc 2010; 42: 3001. 
15. Asleh R, Clavell AL, Pereira NL, et al. Incidence of malignancies in patients treated with sirolimus following heart transplantation. J Am Coll Cardiol 2019; 73: 2676.

16. Rogers HW, Weinstock MA, Feldman SR, Coldiron BM. Incidence estimate of nonmelanoma skin cancer (Keratinocyte Carcinomas) in the US Population, 2012. JAMA Dermatol 2015; 151: 1081 .

17. O'Reilly Zwald F, Brown M. Skin cancer in solid organ transplant recipients: advances in therapy and management. J Am Acad Dermatol 2011; 65: 253.

18. Garrett GL, Blanc PD, Boscardin J, et al. Incidence of and risk factors for skin cancer in organ transplant recipients in the United States. JAMA Dermatol 2017; 153: 296.

19. Wong MCS, Lao XQ, Ho KF, Goggins WB, Tse SLA. Incidence and mortality of lung cancer: global trends and association with socioeconomic status. Sci Rep 2017; 7(14300): 19.

20. Dix D. Human carcinogenesis: the role of age and gender. Anticancer Res 2019; 39: 4385.

21. Momper JD, Misel ML, McKay DB. Sex differences in transplantation. Transplant Rev 2017; 31: 145.

22. Magee MH, Blum RA, Lates CD, Jusko WJ. Prednisolone pharmacokinetics and pharmacodynamics in relation to sex and race. J Clin Pharmacol 2001; 41: 1180.

23. Morissette P, Albert C, Busque S, St-Louis G, Vinet B. In vivo higher glucuronidation of mycophenolic acid in male than in female recipients of a cadaveric kidney allograft and under immunosuppressive therapy with mycophenolate mofetil. Ther Drug Monit 2001; 23: 520.

24. Costanzo MR, Dipchand A, Starling R, et al. The international society of heart and lung transplantation guidelines for the care of heart transplant recipients. J Heart Lung Transplant 2010; 29: 914

25. Hojo M, Morimoto T, Maluccio M, et al. Cyclosporine induces cancer progression by a cellautonomous mechanism. Nature 1999; 397: 530.

26. Henry ML. Cyclosporine and tacrolimus (FK506): A comparison of efficacy and safety profiles. Clin Transplant 1999; 13: 209.

27. O'Neill JO, Edwards LB, Taylor DO. Mycophenolate Mofetil and risk of developing malignancy after orthotopic heart transplantation: analysis of the transplant registry of the International Society for Heart and Lung Transplantation. J Hear Lung Transplant. 2006; 25: 1186.

28. Carbone D. Smoking and cancer. Am J Med 1992; 93: S13.

29. Heart disease and cancer risk may be linked: American Heart Association scientific sessions, poster presentation Mo3058 - ScienceDaily [Internet]. [cited 2019 Dec 19]. Available from: https://www.sciencedaily.com/releases/2019/11/191111084923.htm.

30. Acuna SA, Fernandes KA, Daly C, et al. Cancer mortality among recipients of solid-organ transplantation in Ontario, Canada. JAMA Oncol 2016; 2: 463.

31. Yang Y, Wang G, He J, et al. Gender differences in colorectal cancer survival: a metaanalysis. Int J Cancer 2017; 141: 1942

32. Sakurai H, Asamura H, Goya T, et al. Survival differences by gender for resected non-small cell lung cancer: a retrospective analysis of 12,509 cases in a Japanese Lung Cancer Registry study. J Thorac Oncol 2010; 5: 1594.

33. Acuna SA, Huang JW, Scott AL, et al. Cancer screening recommendations for solid organ transplant recipients: a systematic review of clinical practice guidelines. Am J Transplant 2017; 17: 103. 